\title{
Analysis of Space-Time Coding in Correlated Fading Channels
}

\author{
Ahmadreza Hedayat, Student Member, IEEE, Harsh Shah, and Aria Nosratinia, Senior Member, IEEE
}

\begin{abstract}
Antenna spacing and the properties of a scattering environment can create correlation between channel coefficients. Temporal correlation between fading coefficients may also be present, because one may be unable or unwilling to fully interleave the channel symbols. This paper presents a comprehensive analysis of multiple-input multiple-output systems under correlated fading. We calculate pairwise-error-probability (PEP) expressions under quasi-static fading, fast fading, block fading, as well as arbitrarily temporally correlated fading, under Rayleigh and Rician conditions. We use the PEP expressions to calculate union bounds on the performance of trellis space-time codes, super orthogonal space-time codes, linear-dispersion codes, and diagonal algebraic space-time codes.
\end{abstract}

Index Terms-Correlated channels, diversity, fading channels, MIMO signaling, pairwise error probability, space-time codes, uniform error property.

\section{INTRODUCTION}

I N A multiple-antenna system, insufficient antenna spacing, angle spread, or the lack of rich scattering may cause spatial correlation between antennas. Fading coefficients may also exhibit temporal correlations. This paper studies the performance of multiple-input multiple-output (MIMO) radio systems in correlated fading channels. We begin by calculating pairwiseerror-probability (PEP) expressions in a variety of scenarios, including quasi-static fading, fast fading, block-fading, general Rayleigh fading, and Rician fading. We consider spatially correlated fading on the transmit or receive side (or both), as well as combined spatially and temporally correlated fading. We then use the PEPs to develop union bounds for a variety of MIMO signaling scenarios. In particular, we provide bounds for trellis space-time codes [1], superorthogonal space-time (SOSTT) codes [2], linear-dispersion (LD) codes [3], and diagonal algebraic space-time (DAST) codes [4].

A brief history of past work in this area is as follows. Damen et al. [5] studied, via simulations, the effect of parameters such as angle spread and Rician factor on the performance of various space-time signaling and detection schemes. Bölcskei and Paulraj [6] studied the quasi-static channel and found Chernoff bounds that in the high-SNR regime link the diversity to the rank and eigenvalues of the correlation matri-

Manuscript received October 29, 2003; revised October 26, 2004; accepted November 1, 2004.The editor coordinating tha review of this paper and approving it for publication is M. Uysal. This paper was supported in part by the National Science Foundation under Grant CCR-9985171.

The authors are with the Department of Electrical Engineering, University of Texas at Dallas, Richardson, TX 75083 USA (e-mail: hedayat@utdallas.edu; hps031000@utdallas.edu; aria@utdallas.edu).

Digital Object Identifier 10.1109/TWC.2005.858338 ces. Their analysis yields expressions that are equivalent to, but different from, the ones we provide in this paper in the special case of the quasi-static channel. In another work [7], Bölcskei et al. studied the performance of space-frequency coded MIMO-orthogonal frequency-division multiplexing (OFDM) for frequency-selective Rician channels. Uysal and Georghiades [8] derived PEP expressions for transmit-antenna correlation (with no receive correlation or temporal correlation). Dogandzić [9] gives PEP expressions with spatial correlation, but does not consider temporal correlation.

This work augments the existing literature in the following ways. First, instead of focusing on Chernoff bounds, we derive exact expressions in a relatively straightforward manner. The exact expressions can replace Monte Carlo simulations, and thus, are helpful in large-scale simulations of wireless networks. Also, our analysis covers a wider range of channel conditions (e.g., joint spatio-temporal correlation), as well as application to a number of MIMO signaling schemes. We also explore the uniform error property (UEP) of codes in correlated channels and show that UEP may be lost if there is transmit-side antenna correlation.

Throughout this paper, we use the following notation. Matrices are shown in boldface uppercase letters, e.g., $\mathbf{A}$, and vectors are shown in boldface lowercase, e.g., x. The Hermitian, the determinant, and the Frobenius norm of $\mathbf{A}$ are denoted by $\mathbf{A}^{\mathrm{H}},|\mathbf{A}|$, and $\|\mathbf{A}\|$, respectively. The vectorizing operator $\operatorname{vec}(\mathbf{A})$ stacks the columns of the matrix $\mathbf{A}$ in a column vector. The Kronecker product of $\mathbf{A}$ and $\mathbf{B}$ is shown as $\mathbf{A} \otimes \mathbf{B}$.

\section{SySTEM MOdEL}

We consider an MIMO system with $n_{\mathrm{T}}$ transmit and $n_{\mathrm{R}}$ receive antennas. The binary data are encoded and modulated by a space-time encoder. Let $\mathbf{x}_{n}=\left[\begin{array}{lll}x_{1}^{(n)} & \cdots & x_{n_{\mathrm{T}}}^{(n)}\end{array}\right]^{T}$ denote the transmitted signal vector in the $n$th time interval. Let $\mathbf{H}_{n}=\left\{h_{j i}^{(n)}\right\}$ be the $n_{\mathrm{R}} \times n_{\mathrm{T}}$ channel matrix at time $n$, where each entry $h_{j i}$ is the channel gain between transmit antenna $i$ and receive antenna $j$. The channel gains are assumed to be circularly symmetric complex Gaussian random variables, so their magnitude exhibits a Rayleigh distribution. The receivedsignal vector at time $n$ is given by $\mathbf{r}_{n}=\sqrt{E_{\mathrm{s}}} \mathbf{H}_{n} \mathbf{x}_{n}+\mathbf{z}_{n}$, where $\mathbf{z}$ is an $n_{\mathrm{R}} \times 1$ independent identically distributed (i.i.d.) zero-mean Gaussian noise vector and $E_{\mathrm{s}}=\mathcal{E}\left(\|\mathbf{x}\|^{2}\right) / n_{\mathrm{T}}$ is the symbol energy. We assume that the receiver has perfect knowledge of the channel matrix and performs coherent detection. The maximum-likelihood (ML) metric is given by $m(\mathbf{r}, \mathbf{x})=\sum_{n=1}^{N}\left\|\mathbf{r}_{n}-\sqrt{E_{\mathrm{s}}} \mathbf{H}_{n} \mathbf{x}_{n}\right\|^{2}$, where $N$ is the total 
frame (codeword) length, $\mathbf{r}$ is the received signal in this period, and $\mathrm{x}$ is a given codeword in the same period.

\section{Performance Analysis}

The overall error rates can be approximated via the union bound, e.g., for the frame error rate

$$
P_{e} \leq \sum_{\mathbf{x}} P(\mathbf{x}) \sum_{\hat{\mathbf{x}} \neq \mathbf{x}} \mathbf{P}(\mathbf{x} \rightarrow \hat{\mathbf{x}})
$$

where $P(\mathbf{x})$ is the probability of codeword $\mathbf{x}$ (assumed uniform $)$ and $P(\mathbf{x} \rightarrow \hat{\mathbf{x}})$ is the so-called PEP, i.e., the probability that a codeword $\mathbf{x}$ is decoded as $\hat{\mathbf{x}}$. In this section, we concentrate on the development of PEP expressions under a variety of correlated channel conditions.

Using the methods originated by Craig [10] and widely applied by Simon and Alouini [11], we first calculate the conditional PEP and then integrate over channel gain coefficients. An error $\mathbf{x} \rightarrow \hat{\mathbf{x}}$ occurs when $\left\|\mathbf{r}-\sqrt{E_{\mathrm{s}}} \mathbf{H x}\right\|>$ $\left\|\mathbf{r}-\sqrt{E_{\mathrm{s}}} \mathbf{H} \hat{\mathbf{x}}\right\|$, or equivalently, the noise vector $\mathbf{z}$ must be such that $\Re\left\{\sqrt{E_{\mathrm{s}}} \mathbf{z} \mathbf{H}^{*}(\hat{\mathbf{x}}-\mathbf{x})^{*}\right\}>E_{\mathrm{s}} / 2\|\mathbf{H}(\mathbf{x}-\hat{\mathbf{x}})\|^{2}$. Denoting the codeword differences by $\mathbf{e}_{n}=\mathbf{x}_{n}-\hat{\mathbf{x}}_{n}$, the PEP for a given channel realization is [11]

$$
P(\mathbf{x} \rightarrow \hat{\mathbf{x}} \mid \mathbf{H})=Q\left(\sqrt{\frac{E_{\mathrm{s}}}{2 N_{0}} \sum_{n=1}^{N}\left\|\mathbf{H}_{n} \mathbf{e}_{n}\right\|^{2}}\right)
$$

where $N_{0}$ is the one-sided noise-power spectral density. Now, use the alternative integral expression of $Q$-function [10], [11]

$$
Q(x)=\frac{1}{\pi} \int_{0}^{\frac{\pi}{2}} \exp \left(-\frac{x^{2}}{2 \sin ^{2} \theta}\right) d \theta
$$

and subsequently integrate over the randomness of the channel coefficients to obtain the unconditional PEP

$$
\begin{aligned}
P(\mathbf{x} \rightarrow \hat{\mathbf{x}} \mid \mathbf{H}) & =\frac{1}{\pi} \int_{0}^{\frac{\pi}{2}} \exp \left(\frac{-E_{\mathrm{s}}}{4 N_{0} \sin ^{2} \theta} \sum_{n=1}^{N}\left\|\mathbf{H}_{n} \mathbf{e}_{n}\right\|^{2}\right) d \theta \\
P(\mathbf{x} \rightarrow \hat{\mathbf{x}}) & =\frac{1}{\pi} \int_{0}^{\frac{\pi}{2}} \Phi\left(-\frac{E_{\mathrm{s}}}{4 N_{0} \sin ^{2} \theta}\right) d \theta
\end{aligned}
$$

where $\Phi(\cdot)$ is the moment-generating function (MGF) of the random variable $\sum_{n=1}^{N}\left\|\mathbf{H}_{n} \mathbf{e}_{n}\right\|^{2}$. We note that alternative methods for calculating the PEP using MGF do exist, notably the residue method [12]-[14], but Craig's technique is easier and more versatile.

When channel coefficients are i.i.d., the MGF can be decomposed into a product of marginal MGFs and (3) is easily calculated. In the presence of spatial or temporal correlation, however, the MGF cannot be decomposed, and thus, complications arise. The remainder of this section is devoted to providing analytical tools for the calculation of PEP under correlated channel conditions.

We first consider spatially correlated antennas. Correlations can be modeled with an i.i.d. "innovations" channel $\tilde{\mathbf{H}}$ in combination with a linear system. One can think of this as a diagonalization of the correlated channel. The most general form of such a correlation model is constructed using the vectorizing operator $\operatorname{vec}(\cdot)$ as follows:

$$
\operatorname{vec}(\mathbf{H})=\mathbf{R}^{\frac{1}{2}} \operatorname{vec}(\tilde{\mathbf{H}})
$$

where $\mathbf{R}$ is the spatial correlation matrix. A slightly less general, but more useful, model considers correlations on transmit and receive sides separately. Representing the transmit-side correlation matrix as $\mathbf{R}_{\mathrm{Tx}}$ and receive-side correlation matrix as $\mathbf{R}_{\mathrm{Rx}}$, the correlated channel is represented as [15]

$$
\mathbf{H}=\mathbf{R}_{\mathrm{Rx}}^{\frac{1}{2}} \tilde{\mathbf{H}} \mathbf{R}_{\mathrm{Tx}}^{\frac{T}{2}}
$$

where it can be verified that $\mathbf{R}=\mathbf{R}_{\mathrm{Tx}} \otimes \mathbf{R}_{\mathrm{Rx}}$. We use this latter spatial correlation model in our analyses.

\section{A. Quasi-Static Fading With Spatial Correlation}

In quasi-static fading channels, the matrix channel is assumed to be constant over the duration of a codeword, hence $\mathbf{H}_{n}=\mathbf{H}$ for $n=1, \ldots, N$. Denote the difference of two codeword matrices with $\boldsymbol{\Delta}=\left[\mathbf{e}_{1} \cdots \mathbf{e}_{N}\right]$. Then, the argument of the MGF in (3) can be described in terms of $\mathbf{R}_{\mathrm{Rx}}$ and $\mathbf{R}_{\mathrm{Tx}}$

$$
\begin{aligned}
\sum_{n=1}^{N} & \left\|\mathbf{H}_{n} \mathbf{e}_{n}\right\|^{2} \\
& =\|\mathbf{H} \boldsymbol{\Delta}\|^{2} \\
& =\operatorname{tr}\left(\mathbf{H} \boldsymbol{\Delta} \boldsymbol{\Delta}^{\mathrm{H}} \mathbf{H}^{\mathrm{H}}\right) \\
& =\operatorname{vec}\left(\mathbf{H}^{\mathrm{H}}\right)^{\mathrm{H}}\left(\mathbf{I}_{n_{\mathrm{R}}} \otimes \boldsymbol{\Delta} \boldsymbol{\Delta}^{\mathrm{H}}\right) \operatorname{vec}\left(\mathbf{H}^{\mathrm{H}}\right) \\
& =\operatorname{vec}\left(\tilde{\mathbf{H}}^{\mathrm{H}}\right)^{\mathrm{H}} \mathbf{R}_{\mathrm{s}}^{\frac{H}{2}}\left(\mathbf{I}_{n_{\mathrm{R}}} \otimes \boldsymbol{\Delta} \boldsymbol{\Delta}^{\mathrm{H}}\right) \mathbf{R}_{\mathrm{s}}^{\frac{1}{2}} \operatorname{vec}\left(\tilde{\mathbf{H}}^{\mathrm{H}}\right)
\end{aligned}
$$

where $\mathbf{R}_{\mathrm{S}}=\mathbf{R}_{\mathrm{Rx}} \otimes \mathbf{R}_{\mathrm{Tx}}$ is the covariance matrix of $\operatorname{vec}\left(\mathbf{H}^{\mathrm{H}}\right)$ (note the difference with the definition of $\mathbf{R}$ above). We wish to calculate the MGF of the above random variable, which consists of a positive semidefinite quadratic form involving Gaussian vectors $\operatorname{vec}\left(\tilde{\mathbf{H}}^{\mathrm{H}}\right)$.

Fact [16]: Let $\mathbf{A}$ be a Hermitian matrix and $\mathbf{u}$ a circularly symmetric complex Gaussian vector with mean $\overline{\mathbf{u}}$ and covariance matrix $\mathbf{R}_{\mathbf{u}}$. The MGF of the quadratic form $y=\mathbf{u A u} \mathbf{u}^{\mathrm{H}}$ is

$$
\Phi_{y}(s)=\int_{0}^{\infty} \mathrm{e}^{s y} p_{Y}(y) \mathrm{d} y=\frac{\exp \left(s \overline{\mathbf{u}} \mathbf{A}\left(\mathbf{I}-s \mathbf{R}_{\mathbf{u}} \mathbf{A}\right)^{-1} \overline{\mathbf{u}}^{\mathrm{H}}\right)}{\left|\mathbf{I}-s \mathbf{R}_{\mathbf{u}} \mathbf{A}\right|}
$$

where $\mathbf{I}$ is the identity matrix with appropriate size.

Clearly $\mathbf{u}=\operatorname{vec}\left(\tilde{\mathbf{H}}^{\mathrm{H}}\right)^{\mathrm{H}}$ is a zero-mean Gaussian vector with covariance matrix $\mathbf{R}_{\mathbf{u}}=\mathbf{I}_{n_{\mathrm{T}} n_{\mathrm{R}}}$ and $\mathbf{R}_{\mathrm{s}}^{H / 2}\left(I_{n_{\mathrm{R}}} \otimes \boldsymbol{\Delta} \boldsymbol{\Delta}^{\mathrm{H}}\right) \mathbf{R}_{\mathrm{s}}^{1 / 2}$ 
is a Hermitian matrix, hence, using the properties of the Kronecker product [17]

$$
\begin{aligned}
\Phi(s) & =\left|\mathbf{I}_{n_{\mathrm{R}} n_{\mathrm{T}}}-s \mathbf{R}_{\mathrm{s}}^{\frac{H}{2}}\left(\mathbf{I}_{n_{\mathrm{R}}} \otimes \boldsymbol{\Delta} \boldsymbol{\Delta}^{\mathrm{H}}\right) \mathbf{R}_{\mathrm{s}}^{\frac{1}{2}}\right|^{-1} \\
& =\left|\mathbf{I}_{n_{\mathrm{R}} n_{\mathrm{T}}}-s\left(\mathbf{I}_{n_{\mathrm{R}}} \otimes \boldsymbol{\Delta} \boldsymbol{\Delta}^{\mathrm{H}}\right) \mathbf{R}_{\mathrm{s}}\right|^{-1} \\
& =\left|\mathbf{I}_{n_{\mathrm{R}} n_{\mathrm{T}}}-s\left(\mathbf{I}_{n_{\mathrm{R}}} \otimes \boldsymbol{\Delta} \boldsymbol{\Delta}^{\mathrm{H}}\right)\left(\mathbf{R}_{\mathrm{Rx}} \otimes \mathbf{R}_{\mathrm{Tx}}\right)\right|^{-1} \\
& =\left|\mathbf{I}_{n_{\mathrm{R}} n_{\mathrm{T}}}-s \mathbf{R}_{\mathrm{Rx}} \otimes\left(\boldsymbol{\Delta} \boldsymbol{\Delta}^{\mathrm{H}} \mathbf{R}_{\mathrm{Tx}}\right)\right|^{-1} \\
& =\prod_{i=1}^{n_{\mathrm{T}}} \prod_{j=1}^{n_{\mathrm{R}}}\left(1-s \lambda_{j} \mu_{i}\right)^{-1}
\end{aligned}
$$

where $\lambda_{j}$ are the eigenvalues of $\mathbf{R}_{\mathrm{Rx}}$ and $\mu_{i}$ are the eigenvalues of $\boldsymbol{\Delta} \boldsymbol{\Delta}^{\mathrm{H}} \mathbf{R}_{\mathrm{Tx}}$. Therefore, the PEP in the quasi-static case is

$$
\begin{aligned}
P(\mathbf{x} \rightarrow \hat{\mathbf{x}}) & =\frac{1}{\pi} \int_{0}^{\frac{\pi}{2}} \prod_{i=1}^{n_{\mathrm{T}}} \prod_{j=1}^{n_{\mathrm{R}}}\left(1+\frac{E_{\mathrm{s}}}{4 N_{0} \sin ^{2} \theta} \lambda_{j} \mu_{i}\right)^{-1} d \theta \\
& \leq \prod_{i=1}^{n_{\mathrm{T}}} \prod_{j=1}^{n_{\mathrm{R}}}\left(1+\frac{E_{\mathrm{s}}}{4 N_{0}} \lambda_{j} \mu_{i}\right)^{-1} .
\end{aligned}
$$

Equation (10) is the Chernoff bound for the PEP. The special case of the above analysis for the i.i.d. channel has been reported in [8] and [18]. Also, Bölcskei and Paulraj [6] offer an equivalent expression for this Chernoff bound. Liu and Sayeed [19] have reported the Chernoff bound corresponding to $(8)$.

For the high-SNR approximation of the Chernoff bound, substitute (9) in (3) to get

$$
P(\mathbf{x} \rightarrow \hat{\mathbf{x}}) \leq \frac{1}{2}\left(\frac{E_{\mathrm{s}}}{4 N_{0}}\right)^{-r \hat{r}} \prod_{i=1}^{r} \prod_{j=1}^{\hat{r}} \frac{1}{\lambda_{j} \mu_{i}}
$$

where $r=\operatorname{rank}\left(\boldsymbol{\Delta} \boldsymbol{\Delta}^{\mathrm{H}} \mathbf{R}_{\mathrm{Tx}}\right)$ and $\hat{r}=\operatorname{rank}\left(\mathbf{R}_{\mathrm{Rx}}\right)$.

In the high-SNR regime, the quality of a code is usually analyzed via the diversity order and the coding gain [1]. The diversity order of a pair of codewords is the exponent of SNR, i.e., $r \hat{r}=\operatorname{rank}\left(\boldsymbol{\Delta} \boldsymbol{\Delta}^{\mathrm{H}} \mathbf{R}_{\mathrm{Tx}}\right) \cdot \operatorname{rank}\left(\mathbf{R}_{\mathrm{Rx}}\right)$.

\section{B. Fast Fading With Spatial Correlation}

We consider the case where $\mathbf{H}_{n}$ are independent across $n$, but the entries of each matrix $\mathbf{H}_{n}$ are correlated. In this case, the unconditional PEP can be calculated as follows:

$$
P(\mathbf{x} \rightarrow \hat{\mathbf{x}})=\frac{1}{\pi} \int_{0}^{\frac{\pi}{2}} \prod_{n=1}^{N} \Phi_{n}\left(-\frac{E_{\mathrm{s}}}{4 N_{0} \sin ^{2} \theta}\right) d \theta
$$

where $\Phi_{n}(\cdot)$ is the MGF of the random variable $\left\|\mathbf{H}_{n} \mathbf{e}_{n}\right\|^{2}$. Similar to the quasi-static case, we use the concept of a virtual (innovations) channel and a diagonalizing argument to arrive at a useful expression for the PEP as follows:

$$
\begin{aligned}
\Phi_{n}(s) & =\left|\mathbf{I}_{n_{\mathrm{R}} n_{\mathrm{T}}}-s\left(\mathbf{I}_{n_{\mathrm{R}}} \otimes \mathbf{e}_{n} \mathbf{e}_{n}^{\mathrm{H}}\right) \mathbf{R}_{\mathrm{s}}\right|^{-1} \\
& =\left|\mathbf{I}_{n_{\mathrm{R}} n_{\mathrm{T}}}-s \mathbf{R}_{\mathrm{Rx}} \otimes\left(\mathbf{e}_{n} \mathbf{e}_{n}^{\mathrm{H}} \mathbf{R}_{\mathrm{Tx}}\right)\right|^{-1} \\
& =\prod_{j=1}^{n_{\mathrm{R}}}\left(1-s \lambda_{j} \mu_{n}\right)^{-1} .
\end{aligned}
$$

The nonzero eigenvalue of $\mathbf{e}_{n} \mathbf{e}_{n}^{\mathrm{H}} \mathbf{R}_{\mathrm{Tx}}$ is denoted $\mu_{n}$, and $\lambda_{j}$ are the eigenvalues of $\mathbf{R}_{\mathrm{Rx}}$

$$
P(\mathbf{x} \rightarrow \hat{\mathbf{x}})=\frac{1}{\pi} \int_{0}^{\frac{\pi}{2}} \prod_{n=1}^{N} \prod_{j=1}^{n_{\mathrm{R}}}\left(1+\frac{E_{\mathrm{s}} \lambda_{j} \mu_{n}}{4 N_{0} \sin ^{2} \theta}\right)^{-1} d \theta
$$

Unlike the quasi-static case, this equation shows time diversity (when $\boldsymbol{\Delta}$ is nonzero). Interestingly, the overall diversity depends only on the rank of $\mathbf{R}_{\mathrm{Rx}}$, and not that of $\mathbf{R}_{\mathrm{Tx}}$. This is due to the fact that the rank of $\mathbf{e}_{n} \mathbf{e}_{n}^{\mathrm{H}}$ is one, and hence, $\mathbf{e}_{n} \mathbf{e}_{n}^{\mathrm{H}} \mathbf{R}_{\mathrm{Tx}}$ has only one nonzero eigenvalue. In fact, we will see that to exploit full antenna diversity, the channel must remain fixed over $n_{\mathrm{T}}$ symbol durations. Finally, the coding gain depends on the eigenvalues of both $\mathbf{R}_{\mathrm{Rx}}$ and $\mathbf{R}_{\mathrm{Tx}}$. It is possible to obtain a coding-gain expression in this case in a manner similar to [6].

It is insightful to evaluate (14) for the special case where the antennas are uncorrelated. In this case, the nonzero eigenvalue of $\mathbf{e}_{n} \mathbf{e}_{n}^{\mathrm{H}}$ is equal to $\sum_{i=1}^{n_{\mathrm{T}}}\left|x_{i}^{(n)}-\hat{x}_{i}^{(n)}\right|^{2}$ and we have

$$
\Phi_{n}(s)=\left(1-s \sum_{i=1}^{n_{\mathrm{T}}}\left|x_{i}^{(n)}-\hat{x}_{i}^{(n)}\right|^{2}\right)^{-n_{\mathrm{R}}}
$$

Substituting in (12) and setting $s=-E_{\mathrm{s}} / 4 N_{0} \sin ^{2} \theta$ yields a result similar to the one reported in [18].

\section{Block Fading With Spatial Correlation}

The block fading model is a useful approximation for a timevarying fading channel when fading is not fast enough to be represented with a temporally i.i.d. process, but also not slow enough to be well approximated with a quasi-static model. The block fading model has been widely used in the literature. In this model, time is divided into intervals of $M$ symbol transmissions. Fading remains constant over each interval, and is statistically independent from one interval to another. Denoting the length of a codeword with $N$, we assume for simplicity that a codeword length consists of an integer number of blockfading intervals, i.e., $N=K M$.

Using a combination of the methods already described for fast and quasi-static cases, the PEP under block fading can be calculated as

$$
P(\mathbf{x} \rightarrow \hat{\mathbf{x}})=\frac{1}{\pi} \int_{0}^{\frac{\pi}{2}} \prod_{k=1}^{K} \prod_{i=1}^{n_{\mathrm{T}}} \prod_{j=1}^{n_{\mathrm{R}}}\left(1+\frac{E_{\mathrm{s}} \lambda_{j} \mu_{k, i}}{4 N_{0} \sin ^{2} \theta}\right)^{-1} d \theta
$$


where $\boldsymbol{\Delta}_{k}=\left[\begin{array}{lll}\mathbf{e}_{k, 1} & \cdots & \mathbf{e}_{k, M}\end{array}\right]$, and $\mu_{k, i}$ are the eigenvalues of $\boldsymbol{\Delta}_{k} \boldsymbol{\Delta}_{k}^{\mathrm{H}} \mathbf{R}_{\mathrm{Tx}}$. We notice that the time diversity in this case is at most $K$. The rank of $\mathbf{R}_{\mathrm{Rx}}$ affects the diversity directly. But the rank of $\boldsymbol{\Delta}_{k} \boldsymbol{\Delta}_{k}^{\mathrm{H}} \mathbf{R}_{\mathrm{Tx}}$ is equal to $n_{\mathrm{T}}$ only if block size $M \geq$ $n_{\mathrm{T}}$. Thus, in order to achieve full transmit-antenna diversity, the channel must be constant for at least $n_{\mathrm{T}}$ symbol durations. Both $\mathbf{R}_{\mathrm{Rx}}$ and $\mathbf{R}_{\mathrm{Tx}}$ affect the coding gain.

\section{Joint Spatio-Temporal Correlation}

In the previous sections, the channel was modeled either as fast fading (interleaved) or quasi-static. In many cases, due to the size of the codewords or other constraints, one may not be able to use interleaving. In the absence of interleaving, block fading is only an approximation to a slowly fading channel. A more accurate model of channel variations must describe general sample-to-sample correlations. In this section, we consider this general case.

For calculating PEP, we are interested in codeword coordinates where $\mathbf{x}$ and $\hat{\mathbf{x}}$ are not identical. Assume that among the $N$ time indices, the two codewords $\mathbf{x}$ and $\hat{\mathbf{x}}$ are different in the time instances $\left\{k_{1}, \ldots, k_{d}\right\}$. Let the channel matrix at time $k_{i}$ be denoted as $\mathbf{H}_{i}$, and define $\mathcal{H}=\left[\mathbf{H}_{1} \cdots \mathbf{H}_{d}\right]$. Each $\mathbf{H}_{i}$ may be spatially correlated with $\operatorname{vec}\left(\mathbf{H}_{i}\right)$ modeled by a matrix $\mathbf{R}_{\mathrm{s}}=$ $\mathbf{R}_{\mathrm{Tx}} \otimes \mathbf{R}_{\mathrm{Rx}}$. We assume the statistics to be stationary (time invariant), therefore only one spatial correlation matrix suffices. Let $\mathbf{e}_{i}=\mathbf{x}_{k_{i}}-\hat{\mathbf{x}}_{k_{i}}$ and let $\boldsymbol{\Lambda}=\mathbf{e}_{1} \oplus \cdots \oplus \mathbf{e}_{d}$, according to the notation of [17], i.e., $\boldsymbol{\Lambda}$ is the block diagonal matrix whose diagonal elements are vectors $\mathbf{e}_{i}$. The PEP for a given channel realization is

$$
P(\mathbf{x} \rightarrow \hat{\mathbf{x}} \mid \mathbf{H})=\frac{1}{\pi} \int_{0}^{\frac{\pi}{2}} \exp \left(-\frac{E_{\mathrm{s}}}{4 N_{0} \sin ^{2} \theta}\|\mathcal{H} \mathbf{\Lambda}\|^{2}\right) d \theta
$$

thus, the unconditional PEP depends on the MGF of the random variable $\|\mathcal{H} \boldsymbol{\Lambda}\|^{2}$. Assume that the temporal correlation of the channel is modeled by $\mathbf{R}_{t}$, that is, $\mathbf{R}_{t}(i, j)$ is the correlation of the channel between two time instances $i$ and $j$. After some algebra, $\mathcal{H}$ can be described as follows:

$$
\mathcal{H}=\mathbf{R}_{\mathrm{Rx}}^{\frac{1}{2}} \tilde{\mathcal{H}}\left(\mathbf{R}_{t} \otimes \mathbf{R}_{\mathrm{Tx}}\right)^{\frac{T}{2}}
$$

where $\tilde{\mathcal{H}}$ is a $\left(n_{\mathrm{T}} n_{\mathrm{R}}\right) \times d$ matrix with i.i.d. elements. Note that $\operatorname{vec}\left(\mathcal{H}^{\mathrm{H}}\right)=\mathbf{R}^{1 / 2} \operatorname{vec}\left(\tilde{\mathcal{H}}^{\mathrm{H}}\right)$, where $\mathbf{R}=\mathbf{R}_{\mathrm{Rx}} \otimes$ $\mathbf{R}_{t} \otimes \mathbf{R}_{\mathrm{Tx}}$. Using arguments similar to those in Section III-A

$$
\Phi(s)=\left|\mathbf{I}_{d n_{\mathrm{T}} n_{\mathrm{R}}}-s \mathbf{R}_{\mathrm{Rx}} \otimes\left(\boldsymbol{\Lambda} \mathbf{\Lambda}^{\mathrm{H}}\left(\mathbf{R}_{t} \otimes \mathbf{R}_{\mathrm{Tx}}\right)\right)\right|^{-1} .
$$

We can substitute this expression in (3) to obtain the appropriate PEP. High-SNR approximations of the Chernoff bound corresponding to this PEP is

$$
P(\mathbf{x} \rightarrow \hat{\mathbf{x}}) \leq \frac{1}{2}\left(\frac{E_{\mathrm{s}}}{4 N_{0}}\right)^{-r \hat{r}} \prod_{i=1}^{r} \prod_{j=1}^{\hat{r}}\left(\frac{1}{\lambda_{j} \mu_{i}}\right)^{-1}
$$

where the $\mu_{i}$ are the nonzero eigenvalues of $\boldsymbol{\Lambda} \boldsymbol{\Lambda}^{\mathrm{H}}\left(\mathbf{R}_{t} \otimes \mathbf{R}_{\mathrm{Tx}}\right)$. The high-SNR bounds can be used, as usual, for deriving design criteria.

\section{E. Performance Under Rician Fading}

The presence of a line-of-sight component creates a nonzero mean component for the complex Gaussian channel, leading to Rician fading. We denote the mean value of the matrix channel as $\overline{\mathbf{H}}$. The matrix channel can be represented as $\overline{\mathbf{H}}+\mathbf{H}$, where $\mathbf{H}$ is a circularly symmetric complex Gaussian matrix. The innovations of the channel gain coefficients is calculated in the same manner as before to give $\mathbf{R}_{\tilde{\mathbf{H}}}=(1 / 1+K) \mathbf{I}_{n_{\mathrm{T}} n_{\mathrm{R}}}$, where $K$ is the Rician factor.

Recall that the key to the developments for the Rayleigh case was an expression for the MGF of a quadratic form, namely (6). The calculations in the Rician case follow the same general procedure, except that the numerator of the MGF expression, which simplified to unity in the Rayleigh case, will be more complicated in the Rician channel. For the sake of brevity, in the following we omit the details and give only the final PEP expressions.

For the quasi-static case, define $\boldsymbol{\Gamma}=\mathbf{R}_{\mathrm{s}}^{H / 2}\left(\mathbf{I}_{n_{\mathrm{R}}} \otimes \boldsymbol{\Delta} \boldsymbol{\Delta}^{\mathrm{H}}\right)$ $\mathbf{R}_{\mathrm{S}}^{1 / 2}$. Then, the PEP is as represented by (19) found at the bottom of the page.

For the fast-fading case, let $\boldsymbol{\Gamma}_{n}=\mathbf{R}_{\mathrm{s}}^{H / 2}\left(\mathbf{I}_{n_{\mathrm{R}}} \otimes \mathbf{e}_{n} \mathbf{e}_{n}^{\mathrm{H}}\right) \mathbf{R}_{\mathrm{s}}^{1 / 2}$. In this case, the PEP as represented by (20), found at the bottom of the next page.

One may verify the validity of (20) in the special case of an uncorrelated channel, by setting $\boldsymbol{\Gamma}_{n}=\mathbf{I}_{n_{\mathrm{R}}} \otimes \mathbf{e}_{n} \mathbf{e}_{n}^{\mathrm{H}}$, which leads to a result that has been reported in [18].

For the spatially and temporally correlated Rician channel, using existing definitions, the PEP expression is given by (21) and (22), found at the bottom of the next page, where $\boldsymbol{\Gamma}=$ $\mathbf{R}^{H / 2}\left(\mathbf{I}_{n_{\mathrm{R}}} \otimes \boldsymbol{\Lambda} \boldsymbol{\Lambda}^{\mathrm{H}}\right) \mathbf{R}^{1 / 2}$, and $\mathbf{R}=\mathbf{R}_{\mathrm{Rx}} \otimes \mathbf{R}_{t} \otimes \mathbf{R}_{\mathrm{Tx}}$.

In the high-SNR regime, (22) can be written as

$$
\begin{aligned}
P(\mathbf{x} \rightarrow \hat{\mathbf{x}}) \leq & \frac{1}{2}\left(\frac{E_{\mathrm{s}}}{4 N_{0}(1+K)}\right)^{-r \hat{r}} \\
& \prod_{i=1}^{r} \prod_{j=1}^{\hat{r}} \frac{1}{\lambda_{j} \mu_{i}} \exp \left(-(1+K)\|\overline{\mathcal{H}}\|^{2}\right)
\end{aligned}
$$

$$
P(\mathbf{x} \rightarrow \hat{\mathbf{x}})=\frac{1}{\pi} \int_{0}^{\frac{\pi}{2}} \frac{\exp \left(-\frac{E_{\mathrm{s}}}{4 N_{0} \sin ^{2} \theta} \operatorname{vec}\left(\overline{\mathbf{H}}^{\mathrm{H}}\right)^{\mathrm{H}} \boldsymbol{\Gamma}\left(\mathbf{I}_{n_{\mathrm{R}} n_{\mathrm{T}}}+\frac{E_{\mathrm{s}}}{4(1+K) N_{0} \sin ^{2} \theta} \boldsymbol{\Gamma}\right)^{-1} \operatorname{vec}\left(\overline{\mathbf{H}}^{\mathrm{H}}\right)\right)}{\left|\mathbf{I}_{n_{\mathrm{R}} n_{\mathrm{T}}}+\frac{E_{\mathrm{s}}}{4(1+K) N_{0} \sin ^{2} \theta} \boldsymbol{\Gamma}\right|} d \theta
$$


where $\lambda_{j}$ are the eigenvalues of $\mathbf{R}_{\mathrm{Rx}}$, and $\mu_{i}$ are the eigenvalues of $\boldsymbol{\Lambda} \boldsymbol{\Lambda}^{\mathrm{H}} \mathbf{R}_{\mathrm{Tx}}$. It can be shown that $\|\overline{\mathcal{H}}\|^{2}=d n_{\mathrm{T}} n_{\mathrm{R}} K /(1+$ $K)$. Thus, in high SNR, the line-of-sight component offers a fixed coding gain, but no diversity advantage. Note that the result of [7], when specialized to a frequency-flat case, is similar to (24).

\section{F. Insights and Design Issues}

In the high-SNR regime, the quality of a code is usually analyzed via the diversity order and the coding gain [1]. The diversity order of a pair of codewords is the exponent of $\mathrm{SNR}$, i.e., $r \hat{r}=\operatorname{rank}\left(\boldsymbol{\Lambda} \boldsymbol{\Lambda}^{\mathrm{H}}\left(\mathbf{R}_{t} \otimes \mathbf{R}_{\mathrm{Tx}}\right)\right) \cdot \operatorname{rank}\left(\mathbf{R}_{\mathrm{Rx}}\right)$ in (24). Thus, there is loss in diversity if the receive-side correlation is rank deficient. The transmit-side correlation appears via $r \leq \tilde{r}$, where $\tilde{r}=\min \left(\operatorname{rank}\left(\boldsymbol{\Lambda} \boldsymbol{\Lambda}^{\mathrm{H}}\right), \operatorname{rank}\left(\mathbf{R}_{t} \otimes \mathbf{R}_{\mathrm{Tx}}\right)\right)$. If $\mathbf{R}_{\mathrm{Tx}}$ is full rank, then for the quasi-static channel $r \leq \min \left(d, n_{\mathrm{T}}\right)$, and for the fast-fading channel $r \leq d$, where $d=\operatorname{rank}\left(\boldsymbol{\Lambda} \boldsymbol{\Lambda}^{\mathrm{H}}\right)$. The coding gain depends upon $\mid\left(\mathbf{I}_{n_{\mathrm{R}}} \otimes \boldsymbol{\Lambda} \boldsymbol{\Lambda}^{\mathrm{H}}\right)\left(\mathbf{R}_{\mathrm{Rx}} \otimes \mathbf{R}_{t} \otimes\right.$ $\left.\mathbf{R}_{\mathrm{Tx}}\right)\left.|=| \boldsymbol{\Lambda} \boldsymbol{\Lambda}^{\mathrm{H}}\right|^{n_{\mathrm{R}}}\left|\mathbf{R}_{\mathrm{Rx}} \otimes \mathbf{R}_{t} \otimes \mathbf{R}_{\mathrm{Tx}}\right|$. Thus, the code-design criteria can be formalized as follows.

1) Diversity advantage: Maximize the minimum of $\tilde{r}$ over all codeword pairs. If $\mathbf{R}_{\mathrm{Tx}}$ and $\mathbf{R}_{t}$ are full rank, maximize the minimum of $\operatorname{rank}\left(\boldsymbol{\Lambda} \boldsymbol{\Lambda}^{\mathrm{H}}\right)=d$. That is, the minimum symbolwise Hamming distance over all pairs of codewords should be maximized.

2) Coding advantage: Maximize the minimum $\left|\mathbf{\Lambda} \mathbf{\Lambda}^{\mathrm{H}}\right|$ over codeword pairs.

For full-rank $\mathbf{R}_{\mathrm{Tx}}$ and $\mathbf{R}_{t}$, irrespective of the channel correlation, these design criteria are the same as in [1]. We note that as long as the receive- and transmit-side correlation matrices are full rank, there is no loss in diversity, but only in coding gain. A nonidentity temporal correlation matrix also introduces loss in coding gain.

\section{UEP Under TRANSMIT-SIDE CORRELATION}

In general, space-time signaling is nonlinear, thus it does not immediately follow that one can use the all-zero codeword

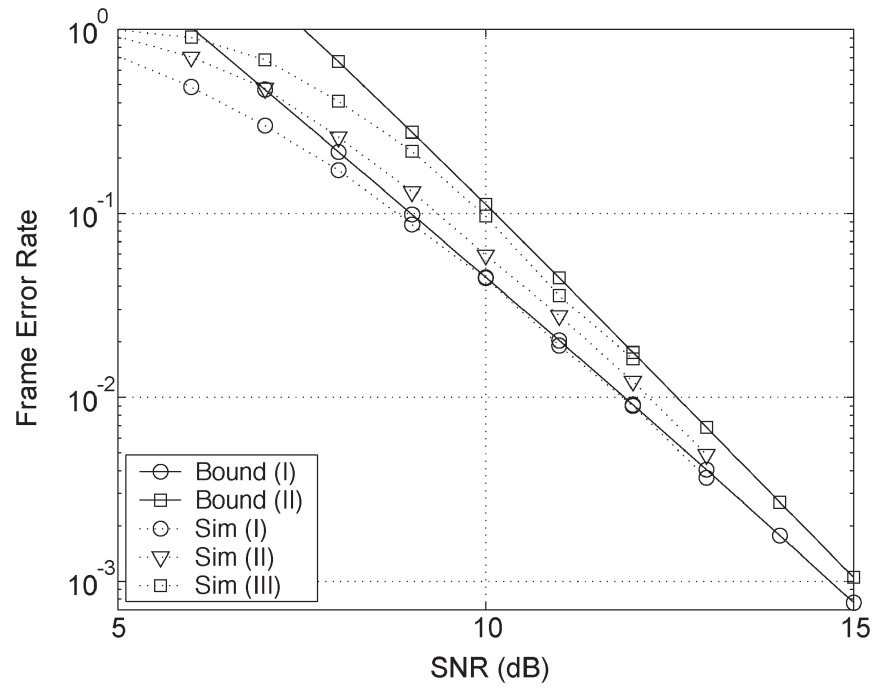

Fig. 1. STT code, 2-Tx, 2-Rx, fast Rayleigh fading, $\rho_{\mathrm{t}}=\rho_{\mathrm{r}}=0.7$.

as reference. However, in many cases, the symmetries in the code (and its corresponding trellis) leads to a condition where the error event probabilities as well as their multiplicities are independent of the transmitted codeword. This class, known as UEP codes [11], includes many of the interesting and practical scenarios. The past analyses have mostly relied on this property.

Interestingly, we found that transmit-side correlation may destroy the UEP property of a code. For example, consider the eight-state QPSK space-time trellis (STT) code of [1], which is ordinarily a UEP code. In Fig. 1, we show the error performance over a spatially correlated channel, for the all-zero codeword with numeral (I), the codeword $02,20,02,20, \ldots$, with numeral (II), and a random codeword with numeral (III). Clearly, the error probability depends on the transmitted codeword, thus, the UEP property has been destroyed.

This phenomenon can be explained as follows. When the channel is spatially uncorrelated, the phase of the received signals are random, so the signals from the multiple transmit antennas may add constructively or destructively with equal

$$
P(\mathbf{x} \rightarrow \hat{\mathbf{x}})=\frac{1}{\pi} \int_{0}^{\frac{\pi}{2}} \prod_{n=1}^{N} \frac{\exp \left(-\frac{E_{\mathrm{s}}}{4 N_{0} \sin ^{2} \theta} \operatorname{vec}\left(\overline{\mathbf{H}}^{\mathrm{H}}\right)^{\mathrm{H}} \boldsymbol{\Gamma}_{n}\left(\mathbf{I}_{n_{\mathrm{R}} n_{\mathrm{T}}}+\frac{E_{\mathrm{s}}}{4(1+K) N_{0} \sin ^{2} \theta} \boldsymbol{\Gamma}_{n}\right)^{-1} \operatorname{vec}\left(\overline{\mathbf{H}}^{\mathrm{H}}\right)\right)}{\left|\mathbf{I}_{n_{\mathrm{R}} n_{\mathrm{T}}}+\frac{E_{\mathrm{s}}}{4(1+K) N_{0} \sin ^{2} \theta} \boldsymbol{\Gamma}_{n}\right|} d \theta
$$

$$
\begin{aligned}
P(\mathbf{x} \rightarrow \hat{\mathbf{x}}) & =\frac{1}{\pi} \int_{0}^{\frac{\pi}{2}} \frac{\exp \left(-\frac{E_{\mathrm{s}}}{4 N_{0} \sin ^{2} \theta} \operatorname{vec}\left(\overline{\mathcal{H}}^{\mathrm{H}}\right)^{\mathrm{H}} \boldsymbol{\Gamma}\left(\mathbf{I}_{d n_{\mathrm{R}} n_{\mathrm{T}}}+\frac{E_{\mathrm{s}}}{4(1+K) N_{0} \sin ^{2} \theta} \boldsymbol{\Gamma}\right)^{-1} \operatorname{vec}\left(\overline{\mathcal{H}}^{\mathrm{H}}\right)\right)}{\left|\mathbf{I}_{d n_{\mathrm{R}} n_{\mathrm{T}}}+\frac{E_{\mathrm{s}}}{4(1+K) N_{0} \sin ^{2} \theta} \boldsymbol{\Gamma}\right|} d \theta \\
& \leq \frac{1}{2} \frac{\exp \left(-\frac{E_{\mathrm{s}}}{4 N_{0}} \operatorname{vec}\left(\overline{\mathcal{H}}^{\mathrm{H}}\right)^{\mathrm{H}} \boldsymbol{\Gamma}\left(\mathbf{I}_{d n_{\mathrm{T}} n_{\mathrm{R}}}+\frac{E_{\mathrm{s}}}{4(1+K) N_{0}} \boldsymbol{\Gamma}\right)^{-1} \operatorname{vec}\left(\overline{\mathcal{H}}^{\mathrm{H}}\right)\right)}{\left|\mathbf{I}_{d n_{\mathrm{T}} n_{\mathrm{R}}}+\frac{E_{\mathrm{s}}}{4 N_{0}(1+K)} \boldsymbol{\Gamma}\right|}
\end{aligned}
$$




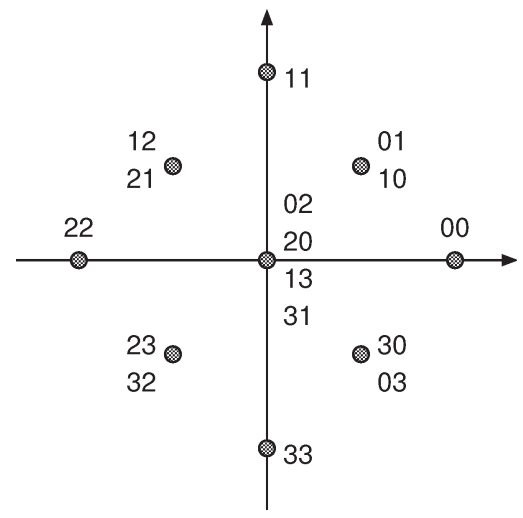

Fig. 2. Received constellation with transmit correlation $\rho_{\mathrm{t}}=1$.

probabilities. But a positive correlation coefficient means that signals add constructively more often than destructively. For example, consider the case of two transmit antennas, one receive antenna, and QPSK modulation, where the modulation symbols are denoted $\{0,1,2,3\}$. In this case, transmission vectors that send the same signal from two antennas (e.g., 00) have an advantage over vectors that send opposite values (e.g., 02 or 13). Worst case codewords are those that are made entirely of these worst case segments. Best case codewords are those made entirely of the same symbol, e.g., the all-zero codeword. A random codeword will contain a mixture of both, and therefore, will fall somewhere in between. ${ }^{1}$

To further demonstrate this effect, we study the case where the two antennas are perfectly correlated. Assuming channel knowledge at the receiver, the equivalent receive constellation is as shown in Fig. 2. Although the minimum distance for all codewords is the same, some codewords (e.g., 00) have a smaller number of nearest neighbors compared to some others (e.g., 02). This effect is further demonstrated in Fig. 3, which depicts the scaled Chernoff bound $P_{\mathrm{sc}}=$ $\left|\mathbf{I}_{n_{\mathrm{T}} n_{\mathrm{R}}}+\left(E_{\mathrm{s}} / 4 N_{0}\right) \boldsymbol{\Delta} \boldsymbol{\Delta}^{\mathrm{H}} \mathbf{R}_{\mathrm{Tx}}\right|^{-1}$ for the PEPs, as well as the corresponding multiplicities. On the left-hand side, we see that when $\rho=0$, the PEP multiplicities are the same for the two codewords 00 and 02 . When $\rho=1$ the multiplicities of $P_{\mathrm{sc}}$ for the two codewords are different, hence the UEP does not hold. A similar effect is seen for other nonzero values of $\rho$.

We note that PEP analysis may not reveal the effect of channel correlation on UEP, because in our examples, the minimum distances are not affected by correlation; only the multiplicity varies. This calls for special care in analyzing codes in the presence of transmit-side correlation.

There are exceptions to the loss of UEP property. For example, the two-state superorthogonal code of [20] with $n_{\mathrm{T}}=2$ and BPSK modulation preserves the UEP property even in the presence of transmit correlation, because all trellis paths transmit space-time block (STB) codewords that are symmetric with respect to spatial correlations; therefore, they are all affected similarly.

\footnotetext{
${ }^{1}$ In the presence of negative correlation, the role of the best case and worst case codewords will be reversed.
}

\section{Application And RESUlts}

In this section, we use the PEP expressions to calculate union bounds for a variety of multiple-antenna signaling and coding schemes, including the space-time trellis (STT) codes [1], SOSTT codes [2], LD codes [3], and DAST block codes [4]. The evaluation of the union bound is possible via either the transfer function or the weight enumerating function. We use the partial weight enumerating function ${ }^{2}$ of the codes for all our results.

Union bounds for the quasi-static channel are often loose [21], due to the fact that each codeword experiences only a single fading coefficient. Malkämaki and Leib [21] proposed a method of limit before averaging to tighten the bounds in quasistatic fading. ${ }^{3}$ Nevertheless, the bounds are still not as tight as the fast-fading case, a fact widely recognized and reported. In the presence of diversity (either temporal or spatial) the union bounds will be tighter and limit before averaging is not needed. This can happen in MIMO channels, thus some of the MIMO quasi-static bounds are tighter than the single-input singleoutput (SISO) case.

In the following figures, dashed lines denote simulations and solid lines denote bounds. In the case of two antennas, $\mathbf{R}_{\mathrm{Tx}}$ and $\mathbf{R}_{\mathrm{Rx}}$ are each fully defined by a single correlation coefficient: $\rho_{\mathrm{t}}$ for the transmit antenna, and $\rho_{\mathrm{r}}$ for the receive antenna. When a higher number of transmit or receive antennas are used, we consider the exponential correlation structure [11] that may correspond to the case of equi-spaced antennas with decaying factor $\rho_{\mathrm{r}}$.

\section{A. STT Codes}

We present the results for STT codes in the context of the eight-state QPSK-STT code in [1], with a spectral efficiency of 2 bits/s/Hz and 130 information symbols per codeword (260 information bits).

Consider the performance of the STT code in quasi-static fading with four receive antennas (see Fig. 4). The results for the uncorrelated quasi-static channel are also included for comparison. We notice that the code has a 2-dB loss due to antenna correlation on the transmit and receive sides.

The union bounds in Fig. 4 are relatively tight, thanks to high diversity. However, the union bound can be loose whenever diversity is low, e.g., if the number of receive antennas is small. For spatially correlated cases, we present the union bound for the worst case reference codeword, as well as for the best codeword. Simulations are shown for both the best codeword or random codeword cases.

\section{B. SOSTT Codes}

Simon and Jafarkhani [20] analyzed the performance of SOSTT codes under spatially and temporally uncorrelated

\footnotetext{
${ }^{2}$ Error events on the order of hundreds were used for the union bounds reported in this paper.

${ }^{3}$ Limit before averaging is relatively straight forward in uncorrelated channels. In correlated channels, limit before averaging requires the multidimensional integration of a nonlinear function that cannot be decomposed, thus, one must use Monte Carlo integration.
} 

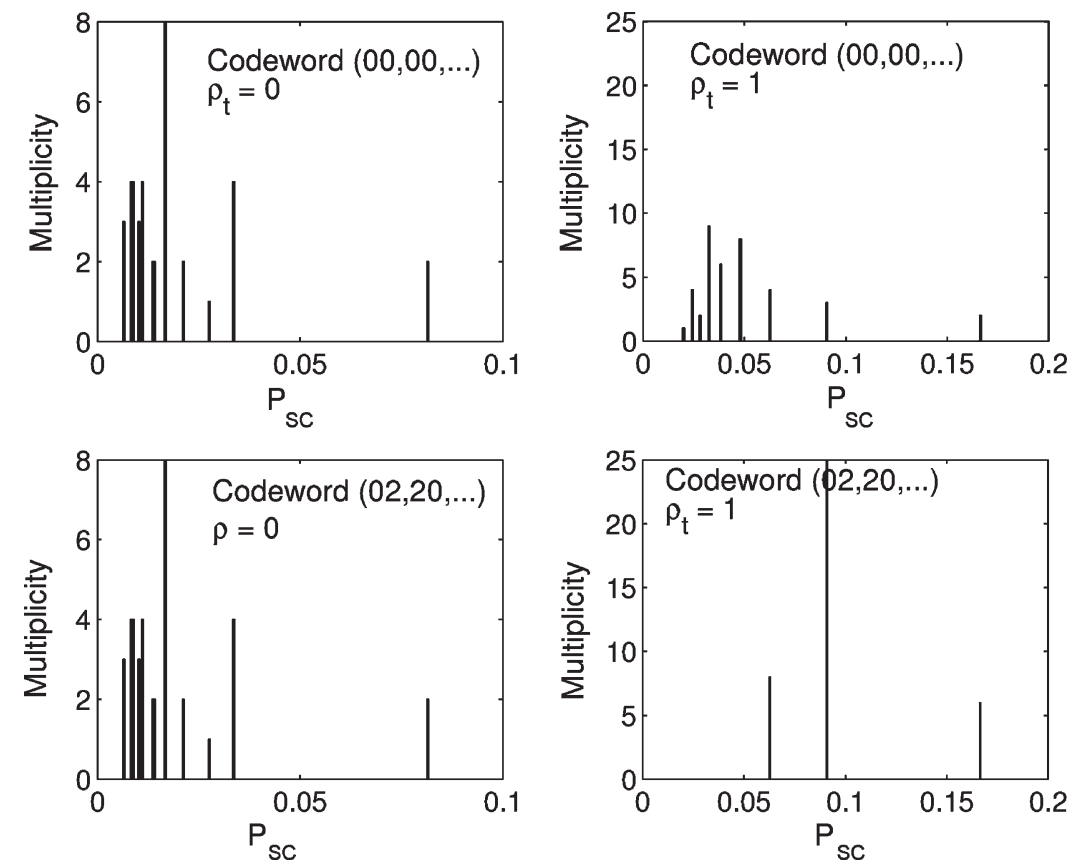

Fig. 3. PEP versus multiplicities for transmitted codewords 00,00 and 02,20 , and $\rho_{\mathrm{t}}=0$, i.e., $\mathbf{R}_{\mathrm{Tx}}=\mathbf{I}$, and $\rho_{\mathrm{t}}=1$, codewords with lengths up to 4 , $\mathrm{SNR}=10 \mathrm{~dB}$.

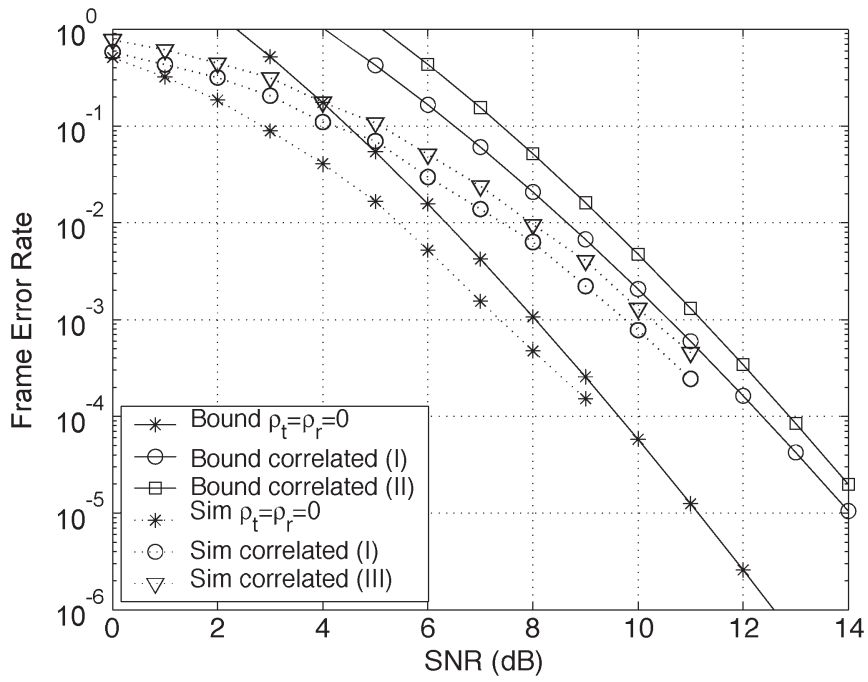

Fig. 4. STT code, 2-Tx, 4-Rx, quasi-static Rayleigh fading, $\rho_{\mathrm{t}}=\rho_{\mathrm{r}}=0.7$.

channel, for which they developed a specialized analysis. Unlike [20], we evaluate the performance of an SOSTT with the same PEP expressions reported in Section III simply by a change of notation, which is as follows. The subscript $n$ in the definition of $\mathbf{x}_{n}$ and $\boldsymbol{\Delta}_{n}$ now stands for the $n$th time-interval with length $n_{\mathrm{T}}$, i.e., the $n$th trellis section. For example, for $n_{\mathrm{T}}=2$, we have

$$
\boldsymbol{\Delta}_{n}=\left(\begin{array}{cc}
x_{n, 1} \mathrm{e}^{j \theta_{n}}-\hat{x}_{n, 1} \mathrm{e}^{j \hat{\theta}_{n}} & -x_{n, 2}^{*} \mathrm{e}^{j \theta_{n}}+\hat{x}_{n, 2}^{*} \mathrm{e}^{j \hat{\theta}_{n}} \\
x_{n, 2}-\hat{x}_{n, 2} & x_{n, 1}^{*}-\hat{x}_{n, 1}^{*}
\end{array}\right)
$$

where $x_{n, 1}$ and $x_{n, 2}$ are the two signals transmitted by the STB code in the $n$th trellis section, and $\theta_{n}$ is the corresponding phase shift, which depends on the encoder state. Using this modification, the PEP in each trellis section (i.e., each block)

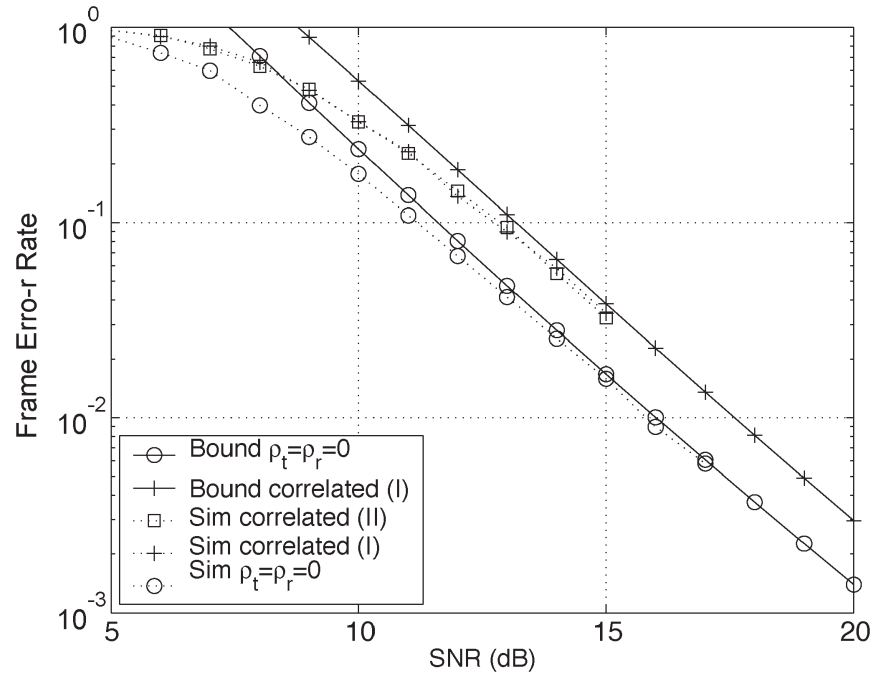

Fig. 5. SOSTT code, 2-Tx, 1-Rx, fast Rayleigh fading, $\rho_{\mathrm{t}}=0.7$.

is given by (15). This change of notation does not affect the results for the quasi-static channel.

For demonstration purposes, we consider the two-state SOSTT code [2] with $n_{\mathrm{T}}=2$ and BPSK modulation, which has a spectral efficiency of $1 \mathrm{bit} / \mathrm{s} / \mathrm{Hz}$. Unlike the STT code in Section V-A, this code preserves its UEP property even when $\mathbf{R}_{\mathrm{Tx}} \neq \mathbf{I}$, because all trellis paths transmit STB codewords that are symmetric with respect to spatial correlations, therefore they are all affected similarly. This may not be true for all block space-time codes, however. The results for fast Rayleigh fading for one receive antenna are shown in Fig. 5. The numeral (I) refers to the all-zero transmitted codeword, and (II) refers to a random codeword. Simulations show no difference, thus confirming UEP property. For comparison, the results for spatially independent fast fading are presented. We see a loss of about 


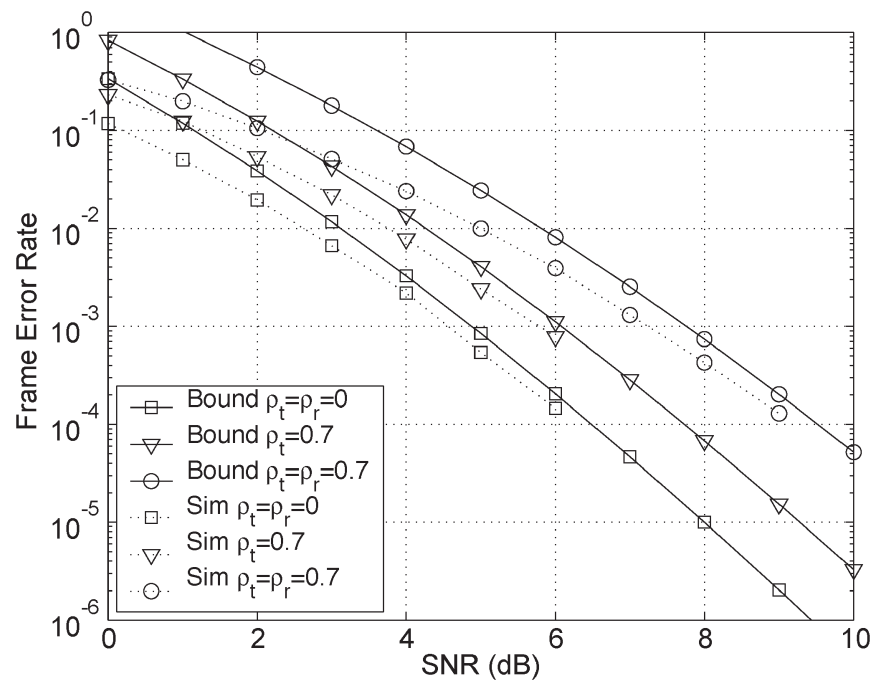

Fig. 6. SOSTT code, 2-Tx, 4-Rx, quasi-static Rayleigh fading, $\rho_{\mathrm{t}}=$ $\rho_{\mathrm{r}}=0.7$.



Fig. 7. SOSTT code, 2-Tx, 4-Rx, temporally correlated Rayleigh fading, $f_{d} T_{\mathrm{s}}=0.01$.

$2 \mathrm{~dB}$ in coding gain due to transmit-side correlation, but no change in diversity.

The performance of the SOSTT code with four receive antennas in a quasi-static spatially correlated channel is shown in Fig. 6 and for the temporally and spatially correlated channel is presented in Fig. 7 .

\section{STB Codes}

Orthogonal STB codes are introduced in [22] as the generalization of the simple Alamouti signaling for $n_{\mathrm{T}}>2$. A related class of block codes with rate-1, entitled quasi-orthogonal codes was later produced by Jafarkhani. It is noteworthy that, for orthogonal STB codes under correlated fading, exact performance analysis (as opposed to bounds) is possible via methods presented in a companion paper [23]. For nonorthogonal block codes, however, the methods in this paper are necessary.

For demonstration purposes, we consider the orthogonal STB code of [22] for $n_{\mathrm{T}}=4$. The time span of this code is 8 , thus the

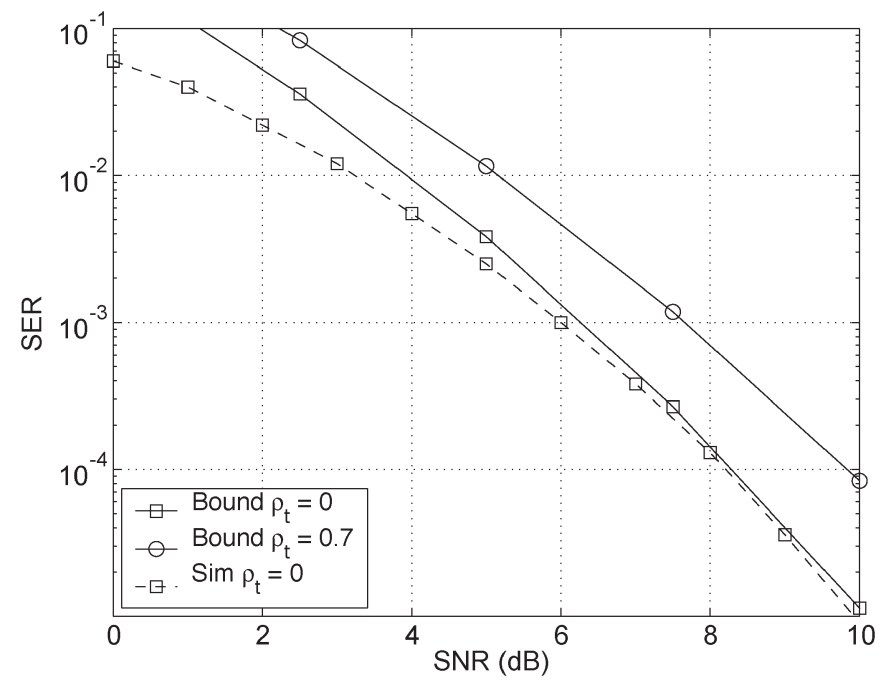

Fig. 8. STB code, 4-Tx, 2-Rx, QPSK, Rayleigh fading.

code has rate $1 / 2$. Using QPSK modulation, this code provides 1-bit/s/Hz spectral efficiency. Fig. 8 presents the symbol error rate of this code for two receive antennas. The bounds are calculated with respect to all codewords. The loss in coding gain is about $1.5 \mathrm{~dB}$ when the transmit antennas are correlated with factor $\rho_{\mathrm{t}}=0.7$.

\section{LD Codes}

LD codes [3] transmit codeword matrices (in time and space) that have the following structure

$$
\mathbf{X}=\sum_{q=1}^{Q}\left(\alpha_{q} A_{q}+j \beta_{q} B_{q}\right)
$$

where $x_{q}=\alpha_{q}+j \beta_{q}$ is a signal chosen from a multitone phase-shift keying (MPSK) or $M$-order quadratic amplitude modulation ( $M$-QAM) modulation, and $A_{q}$ and $B_{q}$ are complex spreading matrices with size $n_{\mathrm{T}} \times T$, where $T$ is the time span of each codeword. We analyze an LD code reported in [3], with $n_{\mathrm{T}}=2, n_{\mathrm{R}}=2, T=2$, and $Q=n_{\mathrm{T}} \times T=4$. We apply the block fading model, i.e., fixed fading coefficient over each block, varying independently between blocks. For QPSK, we have 256 codewords and a rate of $4 \mathrm{bits} / \mathrm{s} / \mathrm{Hz}$. The simulated and analytical frame-error-rate (FER) results of this code are shown in Fig. 9 for uncorrelated as well as spatially correlated antennas. When correlation exists in both the transmit and receive sides, the loss in performance is about $3 \mathrm{~dB}$. We note that LD codes are not UEP, thus, all codeword pairs must be considered for error analysis. For larger constellations (e.g., 16-QAM) this may be cumbersome, and we discovered that considering only adjacent constellation points often gives an acceptable approximation.

\section{E. DAST Codes}

The DAST codeword is given by

$$
\mathbf{X}=\mathcal{H}_{n_{\mathrm{T}}} \operatorname{diag}\left(x_{1}, \ldots, x_{n_{\mathrm{T}}}\right)
$$




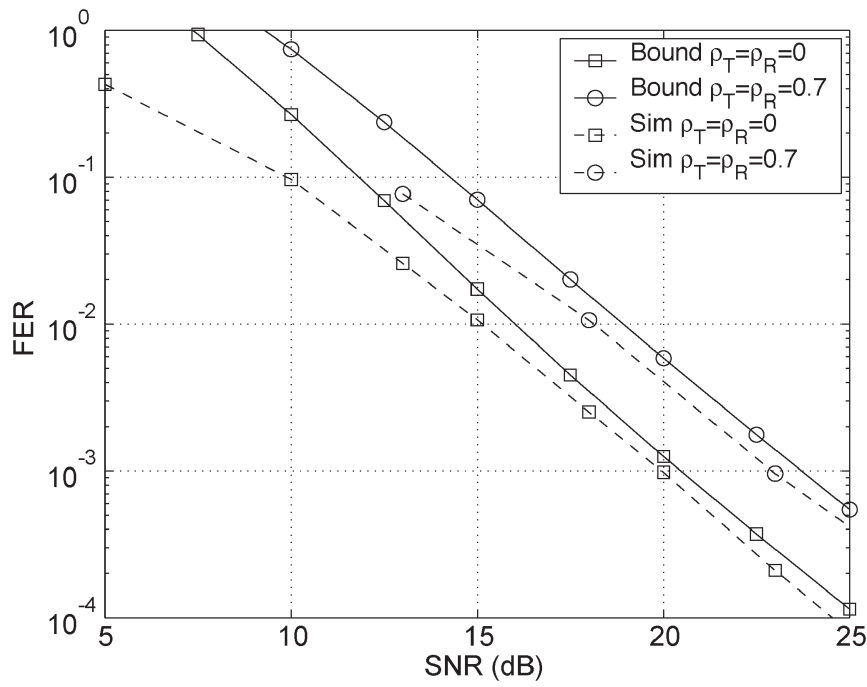

Fig. 9. LD code, 2-Tx, 2-Rx, QPSK, Rayleigh fading.

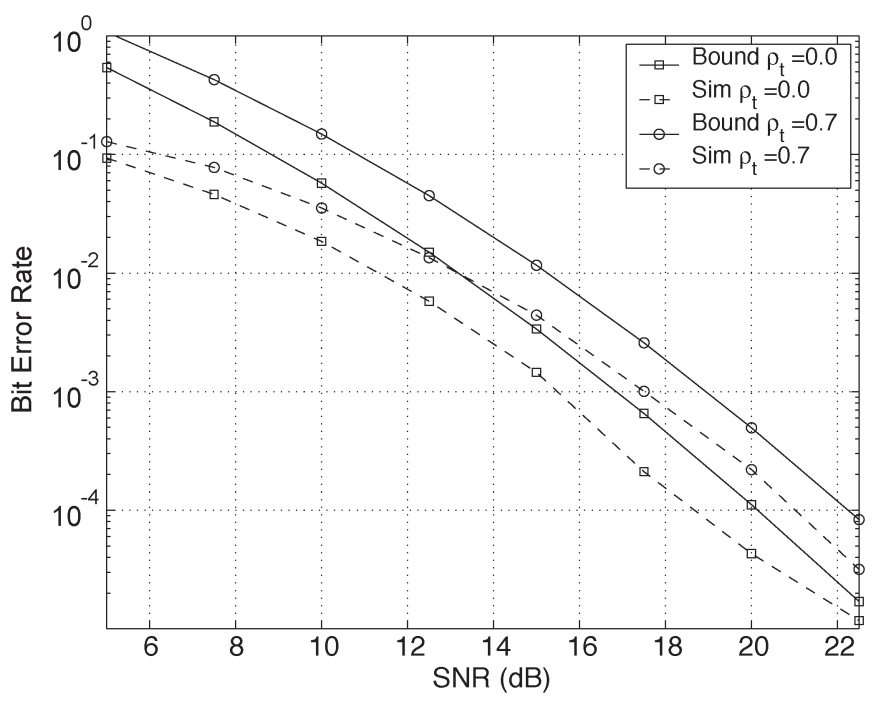

Fig. 10. DAST code, 2-Tx, 2-Rx, QPSK, Rayleigh fading.

where $\left(x_{1}, \ldots, x_{n_{\mathrm{T}}}\right)^{\mathrm{T}}=M_{n_{\mathrm{T}}} \mathbf{a}$, in which a is the information symbol vector, $M_{n_{\mathrm{T}}}$ is a rotation in dimension $n_{\mathrm{T}}$ as defined in [4], and $\mathcal{H}_{n_{\mathrm{T}}}$ is a Hadamard matrix of size $n_{\mathrm{T}} \times n_{\mathrm{T}}$.

We choose a DAST code with $n_{\mathrm{T}}=4$ and $n_{\mathrm{R}}=1$ reported in [4] with QPSK modulation. We again assume a block fading channel, where the fading coefficient is fixed over $n_{\mathrm{T}}$ symbol periods, and varies independently between blocks. The number of codewords is 256 , and the rate is $2 \mathrm{bits} / \mathrm{s} / \mathrm{Hz}$. The simulated and analytical BER for the case are shown in Fig. 10 for uncorrelated as well as spatially correlated antennas. We can see that the loss in performance due to transmit correlation of 0.7 is about $2 \mathrm{~dB}$.

\section{CONCLUSION}

This paper presents a comprehensive analysis of MIMO signaling and coding in the presence of correlated fading. We calculate PEP expressions for a variety of spatially and temporally correlated Rayleigh and Rician channels. Our analysis includes quasi-static and fast fading, block fading, and arbitrarily temporally correlated fading. PEP expressions are then used to generate union bounds on the error probabilities of STT codes, SOSTT codes, and LD codes. Other space-time codes may also be analyzed with the methods presented in this paper.

\section{REFERENCES}

[1] V. Tarokh, N. Seshadri, and A. Calderbank, "Space-time codes for high data rate wireless communication: Performance criteria and code construction," IEEE Trans. Inf. Theory, vol. 44, no. 2, pp. 744-765, Mar. 1998.

[2] H. Jafarkhani and N. Seshadri, "Super-orthogonal space-time trellis codes," IEEE Trans. Inf. Theory, vol. 49, no. 4, pp. 937-950, Apr. 2003.

[3] B. Hassibi and B. Hochwald, "High-rate codes that are linear in space and time," IEEE Trans. Inf. Theory, vol. 48, no. 7, pp. 1804-1824, Jul. 2002.

[4] M. O. Damen, K. Abde-Meraim, and J. C. Belfiore, "Diagonal algebraic space-time block codes," IEEE Trans. Inf. Theory, vol. 48, no. 3, pp. 938 952, Mar. 2002.

[5] M. Damen, A. Abdi, and M. Kaveh, "On the effect of correlated fading on several space-time coding and detection schemes," in Proc. IEEE Vehicular Technology Conf., Atlantic City, NJ, 2001, pp. 13-16.

[6] H. Bölcskei and A. J. Paulraj, "Performance of space-time codes in the presence of spatial fading correlation," in Proc. Asilomar Conf. Signals, Systems and Computers, Pacific Grove, CA, Oct. 2000, pp. 687-693.

[7] H. Bölcskei, M. Borgmann, and A. J. Paulraj, "Impact of the propagation environment on the performance of space-frequency coded MIMOOFDM," IEEE J. Sel. Areas Commun., vol. 21, no. 3, pp. 427-439, Apr. 2003.

[8] M. Uysal and C. Georghiades, "On the error performance analysis of space-time trellis codes," IEEE Trans. Wireless Commun., vol. 3, no. 4, pp. 1118-1123, Jul. 2004.

[9] A. Dogandzić, "Chernoff bounds on pairwise error probabilities of space-time codes," IEEE Trans. Inf. Theory, vol. 49, no. 5, pp. 13271336, May 2003.

[10] J. W. Craig, "A new, simple, and exact result for calculating the probability of error for two-dimensional signal constellations," in Proc. IEEE Military Communications Conf. (MILCOM), McLean, VA, Oct. 1991, pp. 571-575.

[11] M. K. Simon and M. S. Alouini, Digital Communication Over Fading Channels: A Unified Approach to Performance Analysis. New York: Wiley, 2000.

[12] G. Taricco and E. Biglieri, "Exact pairwise error probability of spacetime codes," IEEE Trans. Inf. Theory, vol. 48, no. 2, pp. 510-513, Feb. 2002.

[13] S. Siwamogsatham, M. P. Fitz, and J. H. Grimm, "A new view of performance analysis of transmit diversity schemes in correlated Rayleigh fading," IEEE Trans. Inf. Theory, vol. 48, no. 4, pp. 950-956, Apr. 2002.

[14] M. Brehler and M. K. Varanasi, "Asymptotic error probability analysis of quadratic receivers in Rayleigh fading channels," IEEE Trans. Inf. Theory, vol. 47, no. 6, pp. 2383-2399, Sep. 2001.

[15] C. Chuah, D. Tse, J. Kahn, and R. Valenzuela, "Capacity scaling in MIMO wireless systems under correlated fading," IEEE Trans. Inf. Theory, vol. 48, no. 3, pp. 637-650, Mar. 2002.

[16] G. L. Turin, "The characteristic function of Hermitian quadratic forms in complex normal random variables," Biometrika, vol. 47 , no. 1/2, pp. 199-201, Jun. 1960.

[17] R. Horn and C. R. Johnson, Matrix Analysis, 1st ed. Cambridge, U.K.: Cambridge Univ. Press, 1985.

[18] M. K. Simon, "Evaluation of average bit error probabilities for spacetime coding based on a simpler exact evaluation of pairwise error probability," J. Commun. Netw., vol. 3, no. 3, pp. 257-264, Sep. 2001.

[19] Z. Hong, K. Liu, A. M. Sayeed, and R. Heath, "Spatial multiplexing in correlated fading via the virtual channel representation," in Proc. 40th Annu. Allerton Conf. Comm., Control and Computing, Monticello, IL, Oct. 2002, pp. 161-169.

[20] M. K. Simon and H. Jafarkhani, "Performance evaluation of superorthogonal space-time trellis codes using a moment generating functionbased approach," IEEE Trans. Signal Process., vol. 51, no. 11, pp. 27392751, Nov. 2003.

[21] E. Malkamäki and H. Leib, "Evaluating the performance of convolutional codes over block fading channels," IEEE Trans. Inf. Theory, vol. 45, no. 5, pp. 1643-1646, Jul. 1999. 
[22] V. Tarokh, H. Jafarkhani, and A. Calderbank, "Space-time block codes from orthogonal designs," IEEE Trans. Inf. Theory, vol. 45, no. 5, pp. 1456-1467, Jul. 1999.

[23] H. Shah, A. Hedayat, and A. Nosratinia "Performance of concatenated channel codes and orthogonal space-time block codes," IEEE Trans. Wireless Commun., to be published.



Ahmadreza Hedayat (S'00) received the B.S.E.E. and M.S.E.E. degrees from the University of Tehran, Tehran, Iran, in 1994 and 1997, respectively, and the Ph.D. degree in electrical engineering from the University of Texas at Dallas, in 2004.

From 1995 to 1998, he was a Systems and Hardware Engineer with Pars Telephone Kar. From 1998 to 1999 , he was a Systems and R\&D Engineer with Informatic Services Corporation. His research interests are in wireless and digital communications with emphasis on multiple-antenna systems, channel coding, and cross-layer schemes.

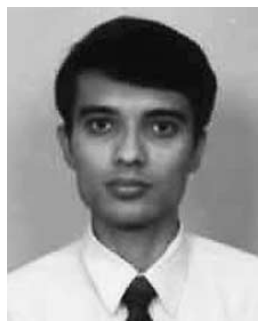

Harsh Shah was born in Ahmedabad, India, in 1978. $\mathrm{He}$ received the B.E. degree in electronics and communications from Gujarat University, Ahmedabad, India, in 2001, the M.S. degree in electrical engineering from University of Texas, Dallas, in 2003, and is currently pursuing the Ph.D. degree in electrical engineering at University of Texas at Dallas.

$\mathrm{He}$ is currently interested in research on digital watermarking, channel coding, and multiple-input multiple-output (MIMO) systems.

Mr. Shah was the recipient of a Kodak Graduate



Aria Nosratinia (M'97-SM'04) received the B.S degree in electrical engineering from the University of Tehran, Tehran, Iran, in 1988, the M.S. degree in electrical engineering from the University of Windsor, Windsor, ON, Canada, in 1991, and the Ph.D. degree in electrical and computer engineering from the University of Illinois at Urbana-Champaign, in 1996.

From 1995 to 1996 , he was with Princeton University, Princeton, NJ. From 1996 to 1999, he was a Visiting Professor and Faculty Fellow at Rice University, Houston, TX. Since July 1999, he has been with the faculty of the University of Texas at Dallas, where he is currently Associate Professor of Electrical Engineering. His research interests are in the broad area of communication and information theory, particularly coding and signal processing for the communication of multimedia signals.

Dr. Nosratinia is Associate Editor for the IEEE TRANSACTIONS ON IMAGE PROCESSING. He was the recipient of the National Science Foundation (NSF) CAREER Award in 2000 and has twice received chapter awards for outstanding service to the IEEE Signal Processing Society.

Fellowship for 2004. 